\title{
Copper beaten skull in X-linked hypophosphataemic rickets
}

\author{
Rapur Ram • Vemuri Chaitanya \\ Vishnubotla Siva Kumar
}

Received: 2 December 2013/Accepted: 30 January 2014/Published online: 25 February 2014

(C) Japanese Society of Nephrology 2014

Keywords Copper beaten skull - Craniosynostosis · Fibroblast growth factor $23 \cdot \mathrm{X}$-linked hypophosphataemic rickets

Convolutional markings could be normal impressions of the gyri on the inner table of the skull, seen predominantly posteriorly. If they are pronounced over the more anterior parts of the skull, then this is referred to as a copper beaten skull (CBK). Silver beaten skull also refers to the same condition. The CBK appearance is typically associated with craniosynostosis (Fig. 1 and supplementary figure). Consequently, the growing brain exerts a continuous pulsatile pressure on the malleable cranium, producing a gyral pattern evidenced on plain skull radiographs. CBK is a consequence of craniosynostosis and not specific for $\mathrm{X}$-linked hypophosphataemic rickets (XLH). In XLH, the levels fibroblast growth factor (FGF) 23 expressed in kidney are elevated, and there is a cross-binding at the cranial sutures of FGF23 with FGF receptor 2 expressed in osteoblasts, thus accounting for association of craniosynostosis and XLH, and this may explain why CBK is seen in XLH.

Conflict of interest The authors have declared that no conflict of interest exists.

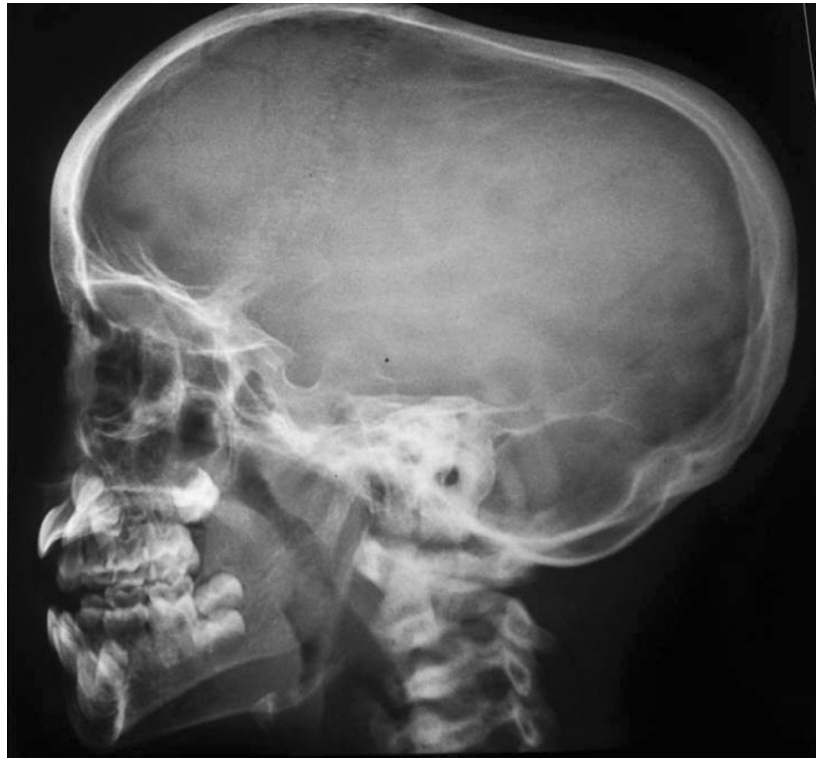

Fig. 1 Lateral radiograph of skull: copper beaten skull
Electronic supplementary material The online version of this article (doi:10.1007/s10157-014-0946-5) contains supplementary material, which is available to authorized users.

R. Ram $(\bowtie) \cdot$ V. Chaitanya · V. Siva Kumar

Department of Nephrology, Sri Venkateswara Institute of

Medical Sciences, Tirupati 517502, India

e-mail: ram_5_1999@yahoo.com 